\title{
SPECTROSCOPY OF SOLIDS
}

\begin{abstract}
CONFERENCE on the Spectroscopy of Solids, held by the Physical Society at the Royal Radar Establishment, Malvern, on May 28 and 29, brought together physicists from many parts of the world engaged in research in this field. Within the past two years, high-resolution infra-red spectroscopy has given a great deal of new information on various processes which lead to optical excitation of electrons from the valence to the conduction band in semi-conductors, and this in turn has led to a better knowledge of the band structure of silicon and germanium and also of a few of the intermetallic semi-conductors. In the opening paper of the conference, R. A. Smith discussed the various types of transition which take place in silicon and germanium, and, in particular, the part played by emission and absorption of phonons. The formation of exciton states was also discussed. These may be associated both with direct transitions in which the crystal momentum does not change, and in which no phonons are involved, and also with indirect transitions involving an appreciable change in crystal momentum and hence the emission or absorption of a phonon. Excitons associated with direct transitions give rise to a line absorption spectrum and those associated with indirect transitions to a band spectrum. Absorption due to both types of exciton formation was reported and discussed (see below).
\end{abstract}

High-resolution absorption spectra corresponding to indirect transitions in germanium, and obtained for a wide range of temperatures extending from $4^{\circ} \mathrm{K}$., have previously been obtained for germanium by G. G. Macfarlane, T. P. McLean, J. E. Quarrington and V. Roberts, of the Royal Radar Establishment, showing absorption due to exciton formation. From these spectra, energies of the longitudinal and transverse acoustic phonons corresponding to the zone-edge in the $\langle 111\rangle$ directions, at which the minima in the conduction band occur, were obtained. These were found to be in good agreement with values obtained by B. N. Brockhouse and K. Iyengar from observations of neutron scattering. Similar measurements for silicon were now reported. From an analysis of these, the phonon energies for momentum values corresponding to the minima in the conduction band in the $\langle 100\rangle$ directions were obtained. Four types of phonon were observed having energies $k \theta$ with $\theta=212^{\circ}, 670^{\circ}, 1,050^{\circ}$ and $1,420^{\circ} \mathrm{K}$. These were interpreted as corresponding, respectively, to the transverse acoustic, longitudinal acoustic, longitudinal optical and transverse optical phonons. The fact that four phonons are observed and not three shows conclusively that the minima in the conduction band for silicon do not lie at the zone-edge as for germanium, since at the zone-edge the longitudinal acoustic and optical phonons have the same energy in the $\langle 100\rangle$ directions. It is estimated that for silicon the minima occur for a crystal momentum of about 0.8 times the value at the zone-edge. Accurate values for the forbidden energy gap in silicon, and for its variation with temperature, were also obtained from these measurements.

Fine structure in the recombination radiation of silicon was reported in a paper by J. R. Haynes, M. Lax and W. Flood, of the Bell Telephone Labor- atories. This radiation was shown to be due to indirect transitions, and those corresponding to emission and absorption of all the four phonons discussed above were identified. Moreover, the phonon energies obtained in this way agree quite well with those obtained from the absorption spectra.

New measurements of the absorption due to the direct transition in germanium were also reported by Macfarlane, McLean, Quarrington and Roberts. These showed a strong line absorption due to the lowest exciton state. Owing to the effect of the Coulomb interaction between the free electron and hole, the form of the spectrum in the region of continuous absorption is considerably modified, and the variation with quantum energy $h v$ is not proportional to $\left(h \nu-\Delta E_{0}\right)^{t}$, as is frequently stated $\left(\Delta E_{0}\right.$ is the forbidden energy gap at the centre of the first Bril. louin zone). The form of the continuous absorption spectrum was measured at high resolution and shown to be in very good agreement with that found theoretically on taking the Coulomb interaction of the free electron and hole into account. An accurate value for $\Delta E_{0}$ is also obtained by this means.

In a theoretical paper on the phonon spectrum of germanium by F. Herman, of the Radio Corporation of America Laboratories, Princeton, forces including the effects of up to fifth nearest neighbours were taken into account. A comparison was made with the most recent observations by Brockhouse and Iyengar, using neutron scattering, of all four branches of the phonon spectrum of germanium.

The effect of strong magnetic fields on the absorption spectrum of germanium and other semi-conductors was described in papers by B. Lax and by S. Zwerdling, of the Lincoln Laboratory, Massachusetts Institute of Technology. Observation of the oscillatory magneto-absorption effect for direct transitions, due to quantization of electrons and holes into Landau levels in the magnetic field, previously reported by these authors, has been extended by use of higher spectral resolution to give the fine structure due to the degeneracy of the valence band of germanium. These observations enable the effective electron mass at the centre of the Brillouin zone to be determined. Magneto-absorption has now also been observed due to the indirect transitions involving emission and absorption of phonons. Observation of absorption in the direct transitions due to exciton formation was also reported; there was some indication of an excited state of the exciton. The variation of the position of the exciton absorption with magnetic field has also been studied.

Infra-red absorption due to impurities in semiconductors was discussed by $\mathrm{E}$. Burstein, of the Naval Research Laboratory, Washington. Recent observations have shown that the excited states of impurities in $p$-type silicon do not give a hydrogen-like spectrum. This effect is due to the degeneracy of the valence band at the centre of the Brillouin zone. The same author also discussed absorption spectra due to lattice vibrations in polar and homopolar crystals. New observations, with increased resolution, of the lattice absorption spectra of silicon were reported by F. A. Johnson, of the Royal Radar Establishment. It is now possible to 
interpret many of the absorption bands in terms of the phonon energies obtained from the indirect inter. band transitions. In most cases, two phonons are involved, but some of the weaker bands require three.

The optical properties of metals were discussed by F. Abeles, of the Institut d'Optique, Paris. Infra-red reflexion spectra have been studied with the view of obtaining information on the effective number of free electrons. Such observations for some of the noble metals and also for the alkali metals were discussed by M. H. Cohen, Cavendish Laboratory, Cambridge, who distinguished between thermal and optical effective masses and showed how the optical data may be used to determine whether the Fermi surface does or does not meet the boundaries of the first Brillouin zone.

Papers on a number of other materials were also presented, including diamond, quartz, and the compounds, InSb, CdTe and $\mathrm{Bi}_{2} \mathrm{Te}_{3}$. These were mainly concerned with observation of absorption and emission spectra. Such spectra have not yet been obtained with the same high resolution, or analysed in the same detail, as for silicon and germanium

The conference was chosen as the occasion for the delivery of the forty-second Guthrie Lecture of the
Physical Society, by Prof. W. E. Lamb, of the Clarendon Laboratory, Oxford, on "Experimental Tests of Quantum Electrodynamics".

During the conference an opportunity was available for delegates to visit the new Solid State Physics Laboratory at the Royal Radar Establishment. This was officially opened on the previous day, May 27, by the Right Hon. Aubrey Jones, Minister of Supply, together with a new laboratory for the study of guided missiles. The latter will be part of the laboratory facilities of the Guided Weapons Depart. ment under Dr. W. H. Penley. The Solid State Physics Laboratory will be included in the Physies Department under Dr. R. A. Smith. This Laboratory has been specially planned for research in the physics of solids and particular care has been taken to ensure very clean conditions. Complete air conditioning is not used throughout the Laboratory, but only in certain sections which are allocated to work in which minute quantities of certain impurities can have disastrous effects on experimental work. The new building also includes a small radiochemical laboratory and fairly extensive facilities for work at liquid-helium and liquid-hydrogen temperatures. Typical examples of the research work already beginning in the new building were demonstrated to those attending the conference. R. A. Sмiтн

\section{THE DIPLOMA IN TECHNOLOGY}

$\mathrm{M}$ ANY who are concerned with the relation of science to industry combine to welcome, as a great educational reform, the institution of courses for the new Diploma in Technology. Candidature is open to science students who have obtained the General Certificate of Education (Advanced Level) or a good Ordinary National Certificate. The courses are mainly held in the top dozen or so colleges of technology, and often on a residential basis. Thus, students from Kent, Cornwall and Cumberland often study together, as at Brunel College, London, and elsewhere.

But obviously, the institution of a number of quite new degree-level courses, in different subjects, takes a good deal of working out, and raises many educational and administrative problems. In order to thrash these out further, the Brunel Staff Association held a national conference on June 20, attended by 180 delegates, being mainly senior members from the colleges concerned. Delegates were welcomed by Dr. J. Topping, principal of the College and himself a member of the National Council for Technological Awards, and by Mr. L. H. Hancock and Mr. E. E. Robinson, the conference chairman and secretary.

The opening paper, by Dr. A. J. Richmond, of the Welsh College of Advanced Technology, Cardiff, outlined the history of this great new educational venture; and the second paper, by Mr. Gordon Smith, of Brunel College, dealt with the industrial training period. While university education is all very well, it often neglects much which the young industrial scientist needs. Consequently, it was decided in 1955 to institute these new four-year courses, and on the sandwich principle. Thus, each student spends alternate periods of six months $(a)$ at college and (b) undergoing industrial training in approved firms. Many companies now cordially welcome the scheme.
Already a large proportion of the sandwich students are industry-based, that is, paid by their firms throughout the year. But the courses are open equally to students direct from a sixth form, with two ad. vanced level and three ordinary level passes in General Certificate of Education examinations. These receive local education authority awards. More than 700 first-year students enrolled during the present session, and more than 1,000 are expected in 1958-59. The Diploma in Technology has already been recognized as the equivalent of a degree by the Burnham Com. mittee.

Several speakers stressed the overloaded nature of many traditional science courses, where the student is expected to memorize far too many points of detail. A more selective, rather than an encyclopædic, approach is required. This comment was heard from as far apart as Mr. T. E. Hall, of Liverpool, with regard to building and civil engineering, and from Dr. E. G. Cowley, of Brighton, with regard to chemistry at both college- and school-level. It is one of the great potential virtues of the Diploma in Technology courses that syllabuses can be com. pletely re-designed from first principles, to cope with this among other points. Mr. H. S. Barlow, of South-East Essex Technical College, however, questioned whether all colleges were seizing these exciting new educational opportunities boldly enough.

Modern methods of teaching and examining were also discussed, together with the general liberalization of approach. It was noted that those who qualify after the four years with a Diploma in Technology will be as useful to their firms as the average graduate is after another year or two of industrial training.

Further particulars about the courses are available from the Secretary, National Council for Technological Awards, 9 Cavendish Square, London, W.1. 